\title{
Cyclic Nucleotide Pharmacology
}

National Cancer Institute

\section{Source}

National Cancer Institute. Cyclic Nucleotide Pharmacology. NCI Thesaurus. Code C18727.

The science concerned with the chemistry, actions, and uses of cyclic nucleoside monophosphates. 